Marquette University

e-Publications@Marquette

$1-2016$

\title{
What Difference do Bystanders Make? The Association of Bystander Involvement with Victim Outcomes in a Community Sample
}

\author{
Sherry L. Hamby \\ Sewanee: The University of the South \\ Marcela C. Weber \\ University of the South, Sewanee \\ John H. Grych \\ Marquette University, john.grych@marquette.edu \\ Victoria Banyard \\ University of New Hampshire
}

Follow this and additional works at: https://epublications.marquette.edu/psych_fac

Part of the Psychology Commons

\section{Recommended Citation}

Hamby, Sherry L.; Weber, Marcela C.; Grych, John H.; and Banyard, Victoria, "What Difference do Bystanders Make? The Association of Bystander Involvement with Victim Outcomes in a Community Sample" (2016). Psychology Faculty Research and Publications. 255.

https://epublications.marquette.edu/psych_fac/255 
Marquette University

e-Publications@Marquette

\title{
Psychology Faculty Research and Publications/College of Arts and Sciences
}

This paper is NOT THE PUBLISHED VERSION; but the author's final, peer-reviewed manuscript. The published version may be accessed by following the link in the citation below.

Psychology of Violence, Vol. 6, No. 1 (January 2016): 91-102. DOI. This article is (C) American Psychological Association. and permission has been granted for this version to appear in $\underline{\mathrm{e}}-$ Publications@Marquette. American Psychological Association. does not grant permission for this article to be further copied/distributed or hosted elsewhere without the express permission from American Psychological Association..

\section{What Difference Do Bystanders Make? The Association of Bystander Involvement With Victim Outcomes in a Community Sample}

\author{
Sherry Hamby \\ Department of Psychology, The University of the South; \\ Marcela C. Weber \\ Department of Psychology, The University of the South \\ John Grych \\ Department of Psychology, Marquette University \\ Victoria Banyard \\ Department of Psychology, University of New Hampshire
}

Acknowledgement: Sherry Hamby is now at the Department of Psychology, Appalachian Center for Resilience Research, Sewanee, Tennessee.

This work was made possible through the support of a grant from the John Templeton Foundation. The 
opinions expressed in this article are those of the authors and do not necessarily reflect the views of the John Templeton Foundation.

Note: Maureen McHugh, PhD served as action editor for this article.-SH

The potential for bystanders to reduce the occurrence of violence and minimize harm when it does occur has led to an upsurge in research on prevention and intervention targeting bystanders (see Banyard, 2011) for a review of many recent studies on this topic). Bystanders are individuals who witness criminal behavior or social rule violations; they may act to help the victim, support the perpetrator, or do nothing. Early work in this area focused on identifying conditions under which bystanders choose to help or not, such as the classic "bystander effect" in which bystanders in groups are less likely to help strangers than bystanders who are alone (Latane \& Darley, 1968). Research on bullying and peer victimization of school-age youth has expanded this work by describing the complexity of the roles that bystanders can play, which can include encouraging the perpetrator as well as protecting the victim (Salmivalli, 2010). More recently, bystanders have been identified as potential allies in violence prevention efforts, with programs designed to promote helpful bystander behavior in the context of problems such as school bullying, intimate partner violence, or sexual assault (Banyard, 2008; Banyard, Plante, \& Moynihan, 2004; Swearer, Espelage, Vaillancourt, \& Hymel, 2010). The promise of such programs depends, however, on a more complete understanding of who potential bystanders are and what they do in response to different forms of violence. Despite this interest across multiple subdisciplines of violence research, we still have surprisingly little data on the actual incidence and impact of bystanders for many forms of victimization. What is more, what little we know about bystander behavior comes from studies of college campuses or urban communities. Much less is understood about bystanders in rural settings. The purpose of this study is to examine patterns of bystander presence, action, and safety for an array of peer- and adult-perpetrated physical, emotional, and sexual victimizations in a large rural community sample.

\section{Existing Research on Bystander Patterns}

Given the extensive interest in bystander intervention for preventing violence, there is surprisingly little data available on typical patterns of bystander presence, especially outside of the bullying literature. Most of the existing literature has focused on antecedents and situational characteristics associated with bystanders. For instance, the National Crime Victimization Survey (NCVS) provides some insight into the conditions under which bystanders are more and less likely to intervene in a violent or potentially violent situation (Planty, 2002). NCVS analyses indicate that bystanders are present, on average, at two thirds (66\%) of violent crimes and that their presence is most common during physical assault crimes and least common during sexual assault (Hart \& Miethe, 2008; Planty, 2002). According to Hart and Miethe, public, weaponless physical assaults by strangers at night had the highest rates of bystander presence (83\%), whereas private, weaponless sexual assaults by nonstrangers during the day had the lowest rates of bystander presence (14\%). Other researchers specifically investigated barriers to bystanders helping (Burn, 2009) including the impact that danger or safety concerns by bystanders may have on intervention (Fischer et al., 2011). Bystanders are more likely to help when the incident occurs in public, the perpetrator is a stranger to the victim, or the perpetrator is not using a weapon (Hart \& Miethe, 2008), and are most likely to "hurt" the situation 
(make it worse) in robberies where the victim and the perpetrator knew each other (Hart \& Miethe, 2008).

Work in the peer literature on bullying has also explored the impact of various bystander actions, focusing more on stable roles that bystanders can play than on specific incidents. In the bullying literature, the role of "defender" is roughly comparable with the helpful bystander from the criminology literature. The concept of "reinforcers" is similar to that of a harmful bystander, someone who supports or encourages the perpetrator. However, in the bullying literature the reinforcer or assistant roles more explicitly imply malicious intention (such as cheering on the bully), whereas in the crime literature there could be a variety of reasons for an unhelpful result including lack of skill or bystanders who themselves become hurt (Salmivalli, 2010; Salmivalli, Voeten, \& Poskiparta, 2011). Further, these categories tend to assume relatively static roles among a group of peers who are all in the same classroom or school, and do not consider victimization that occurs in other contexts, such as the home or community. The type of bullying also appears to affect bystander responses. Complex bullying, which involves both physical and verbal bullying, creates a situation in which bystanders are less likely to defend victims (Oh \& Hazler, 2009). This may be because these complex situations pose a greater threat to bystanders than situations with only one type of bullying. Similarly, in a study on peer perceptions of bullying (Lodge \& Frydenberg, 2005), bystanders mentioned fear for their own safety as a main reason for not acting on the victim's behalf. However, less is known about whether harm to bystanders is a common occurrence.

The crime and bullying literatures are similar in that they primarily document offenses between acquaintances. Thus, it is also useful to examine the literature on family violence, which has taken another approach to understanding bystanders, or witnesses, to use the terminology of this subfield. In this literature, specific topics have received the lion's share of attention, such as the behavior of nonoffending parents in cases of abuse (Elliott \& Carnes, 2001) and rates of child witnesses to domestic violence (DeBoard-Lucas \& Grych, 2011; Hamby, Finkelhor, \& Turner, in press; Hamby, Finkelhor, Turner, \& Ormrod, 2011). For example, the National Survey of Children's Exposure to Violence (Hamby et al., 2011) reported that approximately half of children exposed to family violence yelled at the perpetrator to stop and about 1 in 4 called for help. Research on nonoffending parents has emphasized facilitating their intervention on behalf of their children (Corcoran, 2004; Elliott \& Carnes, 2001). Like bullying research, family violence research has taken strides to investigate bystander characteristics such as their relationship to the victim or perpetrator, their actions on behalf of the victim, and bystander safety.

Across the subfields of sexual assault (Burn, 2009; Orchowski, Gidycz, \& Raffle, 2008), bullying (Nickerson, Mele, \& Princiotta, 2008; Oh \& Hazler, 2009), and family violence (Baldry, 2003; Hartley, 2002), research on bystander behavior is typically conducted in urban or suburban settings, rather than rural ones (Edwards, Mattingly, Dixon, \& Banyard, 2014). However, approximately 1 in 6 violent crimes occur in rural settings and thus understanding aspects of rural crime are important (Rennison, Dragiewicz, \& DeKeseredy, 2013). Preliminary research suggests that rural communities may present different barriers and facilitators of bystander action. For example, the lower population density in rural areas may reduce the likelihood of bystander presence. Social network connections may be different than in urban areas and Edwards found collective efficacy to be related to greater bystander 
intervention among a sample of rural young adults who reported taking action when there was risk for relationship violence. Further, drawing from intimate partner violence incident reports to the police in rural areas, Shernock (2005) found that almost half of the bystanders who reported the incident to the police were related to the victim, and that most bystanders in reported cases were not victimized, perhaps because of their close ties. More research is needed to explore such questions. Other research suggests that helping more generally occurs in greater quantity in rural compared to urban settings (Rushton, 1978). This research suggests both that bystanders may be fewer in number but perhaps that those who are present are more likely to act and with positive results. Yet to date research is lacking on bystander presence to a variety of forms of victimization in rural communities and ways bystanders impact victim outcomes. Thus, a second aim of the current research is to explore the role of bystanders in a rural community sample of adolescents and adults.

\section{Gaps in Existing Literature on Bystanders}

Perhaps surprisingly, given the huge interest in teaching youth how to be more active, helpful bystanders (Banyard, Moynihan, Cares, \& Warner, 2014; Banyard et al., 2004), we have relatively little basic epidemiological information about bystanders. These gaps include baseline frequencies about how often witnesses are present at victimization, who witnesses tend to be, how commonly they assist victims, and whether there are any risks to bystanders. Information on danger to bystanders is crucial for policies and interventions advocating that bystanders step in to aid victims, as is information about whether helpful bystanders improve outcomes for victims. Bystander patterns also need to be examined across different types of incidents. The subfields of bullying, sexual violence, family violence, and community crime research are often "siloed" such that paradigms, models, and breakthroughs in each area are not applied to other subfields (Hamby \& Grych, 2013). This disconnect between subfields is apparent in the bystander literature. Existing data has only covered a limited number of offenses, and comparisons across types are even fewer in number. Such information has the potential to be highly policy-relevant. In the present study, we attempt to integrate the subdisciplines of victimization research by examining bystander involvement baseline rates and associations with victim outcomes for peer, caregiver, and other adult-perpetrated victimization.

\section{The Current Study}

We used a victim-centered, incident-specific approach to describe bystander involvement across a range of interpersonal violence situations, including physical, psychological, and sexual assault. We also assessed bystanders' relationship to the victim, bystander safety, and bystander actions affecting the situation. Unlike many previous studies, which typically survey either children or adults, ours has the advantage of sampling both adolescents and adults, as well as the advantage of examining four outcomes for victims associated with bystander involvement: victim fear during the incident, victim injury, whether victims' school or work routines were disrupted, and victims' current mental health symptoms.

Findings from previous studies, particularly the NCVS, led us to hypothesize that bystanders of family violence will more likely be relatives of victims and bystanders of peer violence will most commonly be victims' friends or acquaintances. We hypothesize that (a) the presence of bystanders will be associated with more positive outcomes for victims, (b) for the subset of cases where a bystander was 
present, we further hypothesize that helpful bystanders will be associated with better outcomes than bystanders whose actions aggravate the situation, and (c) we hypothesize that better victim outcomes will be associated with bystander safety, in contrast to incidents involving harm or threat to the bystander.

\section{Method}

\section{Participants}

Participants were 1703 current residents of the rural southeastern United States ages 11 to 70 years old $(M=29.3$ years, $S D=12.3$ ) who completed a broader survey on character development and coping. About one fifth of the sample (19.2\%) were under the age of 18. For the 11- to 17-year-old group the mean age was $14.92(S D=1.72)$, whereas for the 18 and older group the mean age was $32.78(S D=$ 11.19). Slightly more than one third were male (35.0\%) and almost two thirds were female (62.8\%). The participants who indicated their race $(n=1650)$ were $77.5 \%$ European American, non-Hispanic, 10.5\% African American, non-Hispanic, 7.3\% Hispanic, 4.1\% multiracial, 0.9\% American Indian or Alaska Native, non-Hispanic; 0.5\% Asian, non-Hispanic, and 0.4\% Native Hawaiian or Pacific Islander. More than a third of participants (35\%) who reported annual income $(n=1,527)$ had income under $\$ 20,000$ per year, and more than a third (33.5\%) received public assistance such as food stamps or welfare. The median household income was $\$ 30,000$ to $\$ 40,000$ per year.

\section{Procedure}

The survey was administered by the research team as a computer-assisted self-interview with an audio option, using the Snap10 software platform on computer tablets. Most participants (83\%) were recruited from community events, such as local festivals and county fairs and thus volunteered in response to signs and flyers at the festival (precluding our ability to estimate how many people may have seen the flyers and signs and decided not to participate). Surveys for these participants were completed on site during the festivals in a space created for the research. Others were recruited through word of mouth (13\%) and newspaper, mail, or other advertising (4\%) and took the survey either at the university or in their homes. Our broader range of recruitment strategies allowed us to reach segments of the population who are seldom included in psychology research. Although we made every effort to simplify language, offer an easy-to-use interface, and make available the option to participate via oral interview, we observed that limited reading or computer skills kept some interested individuals from participating. Thus, this sample is most representative of community members with at least a 6th grade reading ability and some experience using a computer. Technical problems and time limitations at events also kept some individuals from completing the survey. The overall completion rate was $86 \%$. On average, the survey took 57 minutes to complete and each participant received a $\$ 30$ Walmart gift card and was provided with information on local community resources. All procedures were approved by the IRB of the host institution.

\section{Materials}

The measures included in this study were part of a larger set of questionnaires assessing a wide range of constructs. 
Victimization

The Juvenile Victimization Questionnaire: Key Domains Short Form (JVQ; Finkelhor, Hamby, Ormrod, \& Turner, 2005; Hamby, Finkelhor, Ormrod, \& Turner, 2004) includes 10 forms of direct victimization including verbal, physical, and sexual victimization. Test-retest reliability and construct validity of the JVQ were established in a previous national sample (Finkelhor et al., 2005). Construct validity was demonstrated with significant, moderate correlations with trauma symptoms and test-retest reliability showed an average kappa of .59 with 95\% percent agreement across administrations, which indicated substantial reliability, especially given the very low base rate for some items.

\section{Peer-perpetrated victimization}

Peer-perpetrated victimization was measured with six items. Three questions asked about relational victimization, such as, "During your childhood, did you get scared or feel really bad because kids were calling you names, saying mean things to you, or saying they didn't want you around?" The other three asked about assault by a peer relative, assault by a nonrelated peer, and physical intimidation ("During your childhood, did any kids, even a brother or sister, pick on you by chasing you, grabbing you, or by making you do something you didn't want to do?").

\section{Adult-perpetrated victimization}

The screener question Physical assault by any adult asked, "At any time in your life, did any grown-up ever hit or attack you on purpose?" One screener on victimization in the home assessed psychological and emotional abuse ("When you were a child, did you get scared or feel really bad because grown-ups called you names, said mean things to you, or said they didn't want you?"). The other asked about physical assault by a caregiver ("Not including spanking on your bottom, during your childhood did a grown-up in your life hit you?”).

\section{Sexual victimization}

The sexual assault item included perpetrators of any age, asking "At any time in your life, did someone make you do sexual things you didn't want to?" The screener and follow-ups on sexual assault were excluded for minors (younger than 18 years old). See tables for descriptions of each item.

For each type of victimization, specific questions were asked first in a yes/no format to learn whether this form of victimization had happened to the participant. When they answered yes to any of these questions they were then directed to an additional series of questions that asked about other characteristics of the event. Participants who indicated that a form of victimization had happened multiple times were asked to answer follow-up questions about the most recent time it happened to them. Victimizations from adults included incidents that may have been more recent as well as retrospective reports of victimization in childhood.

\section{Bystander characteristics}

Participants who had experienced a particular incident were asked three follow-up questions about bystanders (and if a participant had multiple incidents of the same type were asked to answer in relation to the most recent incident), which were adapted from Planty (2002). The first asked, "Did any teen or grown-up see what happened to you, besides you and the person who did this?" with response options of family, friend/acquaintance, police, stranger, or no one. The next follow-up asked, "Did anyone who saw what happened (a) Help in any way, (b) Make things worse, (c) Both help and make things worse, or (d) Didn't help and didn't make it worse?" Finally, participants were asked, "Did any 
witness get hurt or threatened?" Follow-ups for caregiver-perpetrated victimizations were excluded for minors taking the survey.

Incident outcomes

For each victimization incident that a participant had experienced, they were asked the JVQ follow-up questions assessing fear ("thinking back to when it happened, how afraid did you feel?") and disruption of routines ("did you miss any days of school, work, or your normal routine because of what happened?"). For items on physical violence, a follow-up question about physical injury ("were you physically hurt when this happened?") was included as well. Follow-ups for caregiver-perpetrated victimizations were excluded for minors taking the survey. The fear question was answered on a 3point scale (not at all, a little, or very afraid) and routine and injury questions were answered "yes" or "no."

Mental health

We selected 10 of the 28 Trauma Symptom Checklist for Children (Briere, 1996) items used in a national survey (Finkelhor, Turner, Ormrod, \& Hamby, 2009) that loaded the strongest onto the single factor revealed by a factor analysis. We used this version because our sample included both youth and adults. The 10 symptoms assessed were loneliness, sadness, irritability, feeling bad, guilt, worry, dissociation, intrusive images, unpleasant memories, and intrusive memories. All 10 questions asked how often the participant had experienced a certain symptom in the past month. For example, to assess loneliness, we asked, "how often have you experienced loneliness in the past month?" Response categories ranged from 1 (often) to 4 (never), such that possible scores range from 10 to 40 and higher scores indicate better mental health.

\section{Results}

\section{Descriptive Characteristics of Bystanders}

Table 1 presents descriptive information about the frequency of victimization, bystander presence, and identity of the bystander for 10 types of victimization. The most common kinds of victimization were peer victimization, especially psychological forms of peer victimization, but even sexual and adultperpetrated physical and psychological victimizations were each reported by approximately 1 in 5 participants. Bystanders were present for the majority of incidents (60\% to $70 \%$ ) for all victimization types except sexual assault, and bystander presence was fairly similar across different forms of physical and psychological victimization. Bystanders of peer victimization were more commonly friends or acquaintances, whereas victimization perpetrated by adults was most commonly witnessed by family members. Strangers and police were rarely present for any forms of victimization. Table 2 presents data regarding the actions of bystanders and whether the bystanders were themselves harmed or threatened. As seen in Table 2, across all victimization types, bystanders who helped or had no impact were more common than bystanders who harmed the situation or both helped and harmed. For the most part, bystanders were rarely harmed. Rates of bystanders who were threatened or harmed during the reported incidents ranged from approximately 1 in 25 bystanders for social exclusion by peers, but did reach 1 in 6 for sexual victimization, although from a statistical point of view the rate of sexual victimization was relatively low and this estimate is based on fewer cases than for other victimization types. 
Table 1

Frequencies of Victimization, Bystander Presence, and Bystander Type Reported for 10 Victimization Types

\begin{tabular}{|c|c|c|c|c|c|c|}
\hline \multirow[b]{2}{*}{ Incident type } & \multirow{2}{*}{$\begin{array}{c}\text { Percent } \\
\text { victimized }\end{array}$} & \multirow{2}{*}{$\begin{array}{l}\text { Percent of incidents } \\
\text { with bystanders }\end{array}$} & \multicolumn{4}{|c|}{ Types of bystanders ( $\%$ of all incidents with bystanders) } \\
\hline & & & Family & Friend or acquaintance & Police & Stranger \\
\hline \multicolumn{7}{|l|}{ Peer-perpetrated victimization } \\
\hline Assault by nonrelated peers $(\%)$ & 49.7 & 69.5 & 23.5 & 67.6 & 2.0 & 6.9 \\
\hline Assault by youth relatives & 37.4 & 62.8 & 65.1 & 28.1 & 1.9 & 4.9 \\
\hline Physical intimidation by peers & 43.4 & 66.0 & 22.0 & 70.4 & 0.9 & 6.8 \\
\hline Relational aggression by peers & 50.0 & 67.3 & 9.1 & 83.6 & 1.9 & 5.5 \\
\hline Social discrediting by peers & 60.0 & 66.2 & 7.1 & 84.3 & 1.4 & 7.2 \\
\hline Social exclusion by peers & 52.3 & 65.8 & 7.8 & 83.8 & 1.3 & 7.1 \\
\hline \multicolumn{7}{|l|}{ Adult-perpetrated victimization } \\
\hline Physical assault by any adult & 22.9 & 59.5 & 64.3 & 28.6 & 2.9 & 4.3 \\
\hline Physical assault by caregiver & 18.4 & 59.8 & 75.4 & 16.4 & 3.7 & 4.5 \\
\hline Emotional abuse by caregiver & 20.7 & 63.4 & 73.0 & 23.6 & 0.6 & 1.9 \\
\hline \multicolumn{7}{|l|}{ Sexual victimization } \\
\hline Sexual assault by anyone & 20.3 & 18.4 & 53.3 & 26.7 & 6.7 & 13.3 \\
\hline
\end{tabular}

Note. Only participants who reported having experienced a victimization type were asked about bystander presence for that victimization type. Bystander type was only asked when participants reported that a bystander was present for a particular victimization type.

Table 1 Frequencies of Victimization, Bystander Presence, and Bystander Type Reported for 10 Victimization Types

\begin{tabular}{|c|c|c|c|c|c|c|}
\hline & & & $\begin{array}{l}\text { Types of } \\
\text { bystanders (\% of } \\
\text { all incidents with } \\
\text { bystanders }\end{array}$ & & & \\
\hline Incident type & $\begin{array}{l}\text { Percent } \\
\text { victimized }\end{array}$ & $\begin{array}{l}\text { Percent of } \\
\text { incidents with } \\
\text { bystanders }\end{array}$ & Family & $\begin{array}{l}\text { Friend or } \\
\text { cquaintance }\end{array}$ & Police & Stranger \\
\hline \multicolumn{7}{|l|}{ Peer-perpetrated victimization } \\
\hline Assault by nonrelated peers (\%) & 49.7 & 69.5 & 23.5 & 67.6 & 2.0 & 6.9 \\
\hline Assault by youth relatives & 37.4 & 62.8 & 65.1 & 28.1 & 1.9 & 4.9 \\
\hline Physical intimidation by peers & 43.4 & 66.0 & 22.0 & 70.4 & 0.9 & 6.8 \\
\hline Relational aggression by peers & 50.0 & 67.3 & 9.1 & 83.6 & 1.9 & 5.5 \\
\hline Social discrediting by peers & 60.0 & 66.2 & 7.1 & 84.3 & 1.4 & 7.2 \\
\hline Social exclusion by peers & 52.3 & 65.8 & 7.8 & 83.8 & 1.3 & 7.1 \\
\hline \multicolumn{7}{|l|}{ Adult-perpetrated victimization } \\
\hline Physical assault by any adult & 22.9 & 59.5 & 64.3 & 28.6 & 2.9 & 4.3 \\
\hline Physical assault by caregiver & 18.4 & 59.8 & 75.4 & 16.4 & 3.7 & 4.5 \\
\hline Emotional abuse by caregiver & 20.7 & 63.4 & 73.0 & 23.6 & 0.6 & 1.9 \\
\hline \multicolumn{7}{|l|}{ Sexual victimization } \\
\hline Sexual assault by anyone & 20.3 & 18.4 & 53.3 & 26.7 & 6.7 & 13.3 \\
\hline
\end{tabular}

Note. Only participants who reported having experienced a victimization type were asked about bystander presence for that victimization type. Bystander type was only asked when participants reported that a bystander was present for a particular victimization type. 
Table 2

Frequencies of Bystanders' Actions and Harm or Threat to Bystanders, Reported for 10 Victimization Types

\begin{tabular}{|c|c|c|c|c|c|}
\hline \multirow[b]{2}{*}{ Incident type } & \multicolumn{4}{|c|}{ How bystanders' actions affected the situation $(\%)$} & \multirow{2}{*}{$\begin{array}{l}\text { Percent of bystanders who } \\
\text { were hurt or threatened }\end{array}$} \\
\hline & Helped & Harmed & Helped \& harmed & No impact & \\
\hline \multicolumn{6}{|l|}{ Peer-perpetrated victimizations } \\
\hline Assault by nonrelated peers $(\%)$ & 55.1 & 6.6 & 12.1 & 26.3 & 9.3 \\
\hline Assault by youth relatives & 50.6 & 5.3 & 10.9 & 33.3 & 5.8 \\
\hline Physical intimidation by peers & 38.6 & 8.4 & 13.2 & 39.7 & 8.8 \\
\hline Relational aggression by peers & 34.7 & 10.1 & 15.7 & 39.5 & 6.8 \\
\hline Social discrediting by peers & 36.1 & 11.6 & 19.5 & 32.8 & 5.6 \\
\hline Social exclusion by peers & 34.7 & 7.6 & 17.0 & 40.7 & 4.0 \\
\hline \multicolumn{6}{|l|}{ Adult-perpetrated victimizations } \\
\hline Physical assault by any adult & 33.0 & 7.8 & 18.0 & 41.3 & 14.4 \\
\hline Physical assault by caregiver & 29.5 & 3.8 & 16.7 & 50.0 & 16.5 \\
\hline Emotional abuse by caregiver & 25.0 & 8.1 & 18.8 & 48.1 & 11.3 \\
\hline \multicolumn{6}{|l|}{ Sexual victimization } \\
\hline Sexual assault by anyone & 27.3 & 9.1 & 13.6 & 50.0 & 17.8 \\
\hline
\end{tabular}

Note. Participants who reported that no bystanders were present were not asked about bystander's actions or harm/threat to bystanders.

Table 2: Frequencies of Bystanders' Actions and Harm or Threat to Bystanders, Reported for 10 Victimization Types

\begin{tabular}{|c|c|c|c|c|c|}
\hline & & $\begin{array}{l}\text { How bystanders' } \\
\text { actions affected } \\
\text { the situation (\%) }\end{array}$ & & & \\
\hline Incident type & Helped & Harmed & $\begin{array}{l}\text { Helped \& } \\
\text { harmed }\end{array}$ & $\begin{array}{l}\text { No } \\
\text { impact }\end{array}$ & $\begin{array}{l}\text { Percent of } \\
\text { bystanders who } \\
\text { were hurt or } \\
\text { threatened }\end{array}$ \\
\hline \multicolumn{6}{|l|}{ Peer-perpetrated victimizations } \\
\hline Assault by nonrelated peers (\%) & 55.1 & 6.6 & 12.1 & 26.3 & 9.3 \\
\hline Assault by youth relatives & 50.6 & 5.3 & 10.9 & 33.3 & 5.8 \\
\hline Physical intimidation by peers & 38.6 & 8.4 & 13.2 & 39.7 & 8.8 \\
\hline Relational aggression by peers & 34.7 & 10.1 & 15.7 & 39.5 & 6.8 \\
\hline Social discrediting by peers & 36.1 & 11.6 & 19.5 & 32.8 & 5.6 \\
\hline Social exclusion by peers & 34.7 & 7.6 & 17.0 & 40.7 & 4.0 \\
\hline \multicolumn{6}{|l|}{ Adult-perpetrated victimizations } \\
\hline Physical assault by any adult & 33.0 & 7.8 & 18.0 & 41.3 & 14.4 \\
\hline Physical assault by caregiver & 29.5 & 3.8 & 16.7 & 50.0 & 16.5 \\
\hline Emotional abuse by caregiver & 25.0 & 8.1 & 18.8 & 48.1 & 11.3 \\
\hline \multicolumn{6}{|l|}{ Sexual victimization } \\
\hline Sexual assault by anyone & 27.3 & 9.1 & 13.6 & 50.0 & 17.8 \\
\hline
\end{tabular}

Note. Participants who reported that no bystanders were present were not asked about bystander's actions or harm/threat to bystanders.

\section{Bystander Presence and Victim Outcomes}

We examined whether the simple presence of witnesses affected outcomes for victims for each of the 10 forms of victimization (see Table 3). We used four indicators of victim outcome: victim fear, victim injury, victim routine disruption, and victim's current mental health. Chi-square tests were used to examine differences in rates of injury and routine disruption by the presence or absence of bystanders and analyses of variance were used for fear and mental health ratings. 
Table 3

Four Outcomes of Victimization as a Function of Bystander Presence for 10 Victimization Types

\begin{tabular}{|c|c|c|c|c|}
\hline Victimization type & Bystander present & Bystander absent & $\eta^{2}$ & $r$ \\
\hline \multicolumn{5}{|l|}{ Peer-perpetrated victimizations } \\
\hline \multicolumn{5}{|l|}{ Assault by nonrelated peer $(n=777)$} \\
\hline Fear (Mean, $S D)$ & $1.62( \pm .68)$ & $1.59( \pm .71)$ & 0.00 & \\
\hline Physically hurt ( $\%$ yes $)^{*}$ & $30.0 \%$ & $21.3 \%$ & & 0.09 \\
\hline Routine disrupted (\% yes) & $10.6 \%$ & $6.4 \%$ & & 0.07 \\
\hline Current mental health (Mean, SD) & $27.7( \pm 7.5)$ & $27.7( \pm 8.2)$ & 0.00 & \\
\hline \multicolumn{5}{|l|}{ Assault by youth relative $(n=589)$} \\
\hline Fear & $1.70( \pm .75)$ & $1.82( \pm .75)$ & 0.01 & \\
\hline Physically hurt ( $\%$ yes) & 29.1 & 25.9 & & 0.03 \\
\hline Routine disrupted & 10.7 & 6.9 & & 0.06 \\
\hline Current mental health & $26.8( \pm 7.4)$ & $27.3( \pm 8.1)$ & 0.00 & \\
\hline \multicolumn{5}{|l|}{ Physical intimidation by peer $(n=695)$} \\
\hline Fear ${ }^{*}$ & $1.87( \pm .73)$ & $2.02( \pm .74)$ & 0.01 & \\
\hline Routine disrupted & 9.9 & 14.2 & & 0.06 \\
\hline Current mental health & $26.8( \pm 7.7)$ & $27.2( \pm 8.0)$ & 0.00 & \\
\hline \multicolumn{5}{|l|}{ Relational aggression by peer $(n=786)$} \\
\hline Fear ${ }^{*}$ & $1.71( \pm .69)$ & $1.82( \pm .73)$ & 0.01 & \\
\hline Routine disrupted & 14.6 & 9.8 & & 0.07 \\
\hline Current mental health & $26.5( \pm 7.8)$ & $27.1( \pm 7.7)$ & 0.00 & \\
\hline \multicolumn{5}{|l|}{ Social discrediting by peer $(n=961)$} \\
\hline Fear & $1.53( \pm .66)$ & $1.53( \pm .65)$ & 0.00 & \\
\hline Routine disrupted ${ }^{* *}$ & 14.1 & 6.6 & & 0.11 \\
\hline Current mental health & $27.3( \pm 7.6)$ & $27.8( \pm 7.8)$ & 0.00 & \\
\hline \multicolumn{5}{|l|}{ Social exclusion by peer $(n=816)$} \\
\hline Fear & $1.43( \pm .60)$ & $1.46( \pm .66)$ & 0.00 & \\
\hline Routine disrupted & 7.9 & 5.4 & & 0.05 \\
\hline Current mental health & $26.8( \pm 7.4)$ & $27.8( \pm 7.6)$ & 0.00 & \\
\hline \multicolumn{5}{|l|}{ Adult-perpetrated victimizations } \\
\hline \multicolumn{5}{|l|}{ Physical assault by any adult $(n=353)$} \\
\hline Fear & $2.25( \pm .79)$ & $2.23( \pm .79)$ & 0.00 & \\
\hline Physically hurt & 51.2 & 40.6 & & 0.11 \\
\hline Routine disrupted & 20.3 & 13.4 & & 0.09 \\
\hline Current mental health & $26.3( \pm 8.0)$ & $25.7( \pm 8.0)$ & 0.00 & \\
\hline \multicolumn{5}{|l|}{ Physical assault by caregiver ( $n=224$ ) } \\
\hline Fear & $2.21( \pm .80)$ & $2.06( \pm .87)$ & 0.01 & \\
\hline Routine disrupted ${ }^{*}$ & 17.3 & 8.0 & & 0.01 \\
\hline Physically hurt & 46.6 & 37.5 & & 0.09 \\
\hline Current mental health & $26.3( \pm 7.8)$ & $26.4( \pm 7.9)$ & 0.00 & \\
\hline \multicolumn{5}{|l|}{ Emotional abuse by caregiver $(n=254)$} \\
\hline Fear ${ }^{*}$ & $1.94( \pm .77)$ & $1.69( \pm .76)$ & 0.02 & \\
\hline Routine disrupted & 7.5 & 3.4 & & 0.08 \\
\hline Current mental health & $25.4( \pm 7.9)$ & $25.0( \pm 8.4)$ & 0.00 & \\
\hline \multicolumn{5}{|l|}{ Sexual victimization } \\
\hline \multicolumn{5}{|l|}{ Sexual assault by anyone $(n=245)$} \\
\hline Fear & $2.51( \pm .69)$ & $2.57( \pm .66)$ & 0.00 & \\
\hline Physically hurt & 32.6 & 30.2 & & 0.02 \\
\hline Routine disrupted ${ }^{*}$ & 33.3 & 18.1 & & 0.15 \\
\hline Current mental health & $24.9( \pm 8.2)$ & $26.3( \pm 7.8)$ & 0.00 & \\
\hline
\end{tabular}

Note. Fear ratings were on a scale from 1 (not afraid) to 3 (very afraid). Injury was only asked about physical and sexual assault. $n$ varies because only participants who reported having experienced a victimization type were asked about bystander presence for that victimization type. Percentages or means with outcomes significantly better than expected by chance are in bold (lower fear, injury, and routine disrupted and higher mental health). Percentages or means with outcomes significantly worse than expected by chance are italicized (higher fear, injury and routine disrupted and lower mental health).

${ }^{*} p<.05 .{ }^{* *} p<.01$. 
Table 3: Four Outcomes of Victimization as a Function of Bystander Presence for 10 Victimization Types

\begin{tabular}{|c|c|c|c|c|}
\hline Victimization type & $\begin{array}{l}\text { Bystander } \\
\text { present }\end{array}$ & Bystander absent & $\eta^{2}$ & $r$ \\
\hline \multicolumn{5}{|l|}{ Peer-perpetrated victimizations } \\
\hline \multicolumn{5}{|l|}{ Assault by nonrelated peer $(n=777)$} \\
\hline Fear (Mean, SD) & $1.62( \pm .68)$ & $1.59( \pm .71)$ & 0.00 & \\
\hline Physically hurt (\% yes) ${ }^{*}$ & $30.0 \%$ & $21.3 \%$ & & 0.09 \\
\hline Routine disrupted (\% yes) & $10.6 \%$ & $6.4 \%$ & & 0.07 \\
\hline Current mental health (Mean, $S D$ ) & $27.7( \pm 7.5)$ & $27.7( \pm 8.2)$ & 0.00 & \\
\hline \multicolumn{5}{|l|}{ Assault by youth relative $(n=589)$} \\
\hline Fear & $1.70( \pm .75)$ & $1.82( \pm .75)$ & 0.01 & \\
\hline Physically hurt (\% yes) & 29.1 & 25.9 & & 0.03 \\
\hline Routine disrupted & 10.7 & 6.9 & & 0.06 \\
\hline Current mental health & $26.8( \pm 7.4)$ & $27.3( \pm 8.1)$ & 0.00 & \\
\hline \multicolumn{5}{|c|}{ Physical intimidation by peer $(n=695)$} \\
\hline Fear $^{*}$ & $1.87( \pm .73)$ & $2.02( \pm .74)$ & 0.01 & \\
\hline Routine disrupted & 9.9 & 14.2 & & 0.06 \\
\hline Current mental health & $26.8( \pm 7.7)$ & $27.2( \pm 8.0)$ & 0.00 & \\
\hline \multicolumn{5}{|c|}{ Relational aggression by peer $(n=786)$} \\
\hline Fear $^{*}$ & $1.71( \pm .69)$ & $1.82( \pm .73)$ & 0.01 & \\
\hline Routine disrupted & 14.6 & 9.8 & & 0.07 \\
\hline Current mental health & $26.5( \pm 7.8)$ & $27.1( \pm 7.7)$ & 0.00 & \\
\hline \multicolumn{5}{|l|}{ Social discrediting by peer $(n=961)$} \\
\hline Fear & $1.53( \pm .66)$ & $1.53( \pm .65)$ & 0.00 & \\
\hline Routine disrupted $^{* *}$ & 14.1 & 6.6 & & 0.11 \\
\hline Current mental health & $27.3( \pm 7.6)$ & $27.8( \pm 7.8)$ & 0.00 & \\
\hline \multicolumn{5}{|l|}{ Social exclusion by peer $(n=816)$} \\
\hline Fear & $1.43( \pm .60)$ & $1.46( \pm .66)$ & 0.00 & \\
\hline Routine disrupted & 7.9 & 5.4 & & 0.05 \\
\hline Current mental health & $26.8( \pm 7.4)$ & $27.8( \pm 7.6)$ & 0.00 & \\
\hline \multicolumn{5}{|l|}{ Adult-perpetrated victimizations } \\
\hline \multicolumn{5}{|c|}{ Physical assault by any adult $(n=353)$} \\
\hline Fear & $2.25( \pm .79)$ & $2.23( \pm .79)$ & 0.00 & \\
\hline Physically hurt & 51.2 & 40.6 & & 0.11 \\
\hline Routine disrupted & 20.3 & 13.4 & & 0.09 \\
\hline Current mental health & $26.3( \pm 8.0)$ & $25.7( \pm 8.0)$ & 0.00 & \\
\hline \multicolumn{5}{|c|}{ Physical assault by caregiver $(n=224)$} \\
\hline Fear & $2.21( \pm .80)$ & $2.06( \pm .87)$ & 0.01 & \\
\hline Routine disrupted $^{*}$ & 17.3 & 8.0 & & 0.01 \\
\hline Physically hurt & 46.6 & 37.5 & & 0.09 \\
\hline Current mental health & $26.3( \pm 7.8)$ & $26.4( \pm 7.9)$ & 0.00 & \\
\hline \multicolumn{5}{|c|}{ Emotional abuse by caregiver $(n=254)$} \\
\hline Fear $^{*}$ & $1.94( \pm .77)$ & $1.69( \pm .76)$ & 0.02 & \\
\hline Routine disrupted & 7.5 & 3.4 & & 0.08 \\
\hline Current mental health & $25.4( \pm 7.9)$ & $25.0( \pm 8.4)$ & 0.00 & \\
\hline \multicolumn{5}{|l|}{$\begin{array}{l}\text { Sexual victimization } \\
\text { Sexual assault by anyone }(n=245)\end{array}$} \\
\hline Fear & $2.51( \pm .69)$ & $2.57( \pm .66)$ & 0.00 & \\
\hline Physically hurt & 32.6 & 30.2 & & 0.02 \\
\hline Routine disrupted $^{*}$ & 33.3 & 18.1 & & 0.15 \\
\hline Current mental health & $24.9( \pm 8.2)$ & $26.3( \pm 7.8)$ & 0.00 & \\
\hline
\end{tabular}

Note. Fear ratings were on a scale from 1 (not afraid) to 3 (very afraid). Injury was only asked about physical and sexual assault. $n$ varies because only participants who reported having experienced a victimization type were asked about 
bystander presence for that victimization type. Percentages or means with outcomes significantly better than expected by chance are in bold (lower fear, injury, and routine disrupted and higher mental health). Percentages or means with outcomes significantly worse than expected by chance are italicized (higher fear, injury and routine disrupted and lower mental health).

$* p<.05 .{ }^{* *} p<$

The findings were mixed for victim impact associated with the presence of a bystander. For peer physical assaults, victims had significantly higher rates of injury when a bystander was present than absent $(p<.05)$, counter to our prediction. In partial support of our hypothesis, fear was lower when bystanders were present versus absent for some forms of victimization: physical intimidation by peer $(p<.05)$, relational aggression by peer $(p<.05)$. Fear was actually higher with bystanders present to emotional abuse by a caregiver $(p<.05)$. Surprisingly, routine disruption was more likely to occur when bystanders were present for some forms of victimization - sexual assault $(p<.05)$, caregiver physical assault $(p<.05)$, and social discrediting by peer $(p<.01)$. This was counter to our prediction and also showed that different outcomes could be affected differently. Finally, current mental health was largely unaffected by bystander presence or absence. Table 3 also presents effect sizes which were in the small to medium range.

\section{Bystander Actions and Victim Outcomes}

We also examined the relationship between the bystander actions and victim outcomes (see Table 4). These analyses are calculated only for the subgroup of participants reporting that a bystander was present. Using the same four indicators of victim outcome, we examined bystander action by rates of victim's physical injury and rates of victim's routine disruption with chi-squares, and examined bystander action by victim's fear level and current mental health with analyses of variance. 
Table 4

Four Outcomes of Victimization as a Function of Bystanders' Actions for 10 Victimization Types

\begin{tabular}{|c|c|c|c|c|c|c|}
\hline \multirow[b]{2}{*}{ Victimization type } & \multicolumn{4}{|c|}{ Bystander's actions } & \multirow[b]{2}{*}{$\eta^{2}$} & \multirow[b]{2}{*}{$r$} \\
\hline & Helped & Harmed & Helped \& harmed & No impact & & \\
\hline \multicolumn{7}{|l|}{ Peer-perpetrated victimization } \\
\hline \multicolumn{7}{|l|}{ Assault by non-related peer $(n=532)$} \\
\hline Fear (Mean, $S D)^{* *}$ & $1.54( \pm .63)$ & $1.92( \pm .73)$ & $1.74( \pm .64)$ & $1.67( \pm .73)$ & 0.02 & \\
\hline Physically hurt ( $\%$ yes) & $26.6 \%$ & $42.9 \%$ & $29.3 \%$ & $33.7 \%$ & & 0.10 \\
\hline Routine disrupted (\% yes) $)^{* *}$ & $7.2 \%$ & $28.6 \%$ & $8.6 \%$ & $13.0 \%$ & & 0.17 \\
\hline Current mental health (Mean, $S D)^{\circ}$ & $28.4( \pm 7.4)$ & $24.1( \pm 8.0)$ & $25.9( \pm 6.7)$ & $27.9( \pm 7.7)$ & 0.02 & \\
\hline \multicolumn{7}{|l|}{ Assault by youth relative $(n=365)$} \\
\hline Fear ${ }^{* *}$ & $1.59( \pm .72)$ & $1.83( \pm .82)$ & $1.70( \pm .67)$ & $1.90( \pm .79)$ & 0.04 & \\
\hline Physically hurt ${ }^{*}$ & 23.9 & 45.8 & 27.3 & 36.2 & & 0.15 \\
\hline Routine disrupted ${ }^{* * *}$ & 6.5 & 39.1 & 4.8 & 14.6 & & 0.27 \\
\hline Current mental health & $27.3( \pm 7.1)$ & $24.3( \pm 7.7)$ & $28.2( \pm 7.0)$ & $26.0( \pm 7.7)$ & 0.02 & \\
\hline \multicolumn{7}{|l|}{ Physical intimidation by peer $(n=453)$} \\
\hline Fear & $1.71( \pm .70)$ & $2.11( \pm .76)$ & $1.81( \pm .66)$ & $1.98( \pm .76)$ & 0.04 & \\
\hline Routine disrupted & $10.3 \%$ & $20.0 \%$ & $19.0 \%$ & $14.6 \%$ & & 0.10 \\
\hline Current mental health"** & $28.26( \pm 6.98)$ & $25.30( \pm 7.35)$ & $22.98( \pm 8.00)$ & $27.00( \pm 7.97)$ & & 0.05 \\
\hline \multicolumn{7}{|l|}{ Relational aggression by peer $(n=527)$} \\
\hline Fear ${ }^{*}$ & $1.61( \pm .67)$ & $1.81( \pm .71)$ & $1.84( \pm .64)$ & $1.73( \pm .72)$ & 0.02 & \\
\hline Routine disrupted ${ }^{* * *}$ & 7.1 & 30.2 & 17.5 & 15.9 & & 0.19 \\
\hline Current mental health"* & $27.7( \pm 7.2)$ & $24.5( \pm 8.2)$ & $24.3( \pm 7.9)$ & $26.8( \pm 7.8)$ & 0.03 & \\
\hline \multicolumn{7}{|l|}{ Social discrediting by peer $(n=631)$} \\
\hline Fear & $1.35( \pm .57)$ & $1.73( \pm .75)$ & $1.73( \pm .67)$ & $1.54( \pm .66)$ & 0.06 & \\
\hline Routine disrupted ${ }^{* *}$ & 6.6 & 33.8 & 20.0 & 12.3 & & 0.24 \\
\hline Current mental health" & $28.4( \pm 7.1)$ & $25.6( \pm 8.3)$ & $26.5( \pm 7.3)$ & $27.1( \pm 7.9)$ & 0.02 & \\
\hline \multicolumn{7}{|l|}{ Social exclusion by peer $(n=536)$} \\
\hline Fear $^{\dagger}$ & $1.33( \pm .56)$ & $1.48( \pm .60)$ & $1.54( \pm .66)$ & $1.44( \pm .60)$ & 0.02 & \\
\hline Routine disrupted & 4.9 & 12.2 & 11.0 & 8.3 & & 0.09 \\
\hline Current mental health & $27.5( \pm 7.0)$ & $24.5( \pm 8.3)$ & $26.2( \pm 7.6)$ & $26.8( \pm 7.4)$ & 0.01 & \\
\hline \multicolumn{7}{|l|}{ Adult-perpetrated victimization } \\
\hline \multicolumn{7}{|l|}{ Physical assault by any adult $(n=206)$} \\
\hline Fear & $2.10( \pm .79)$ & $2.31( \pm .79)$ & $2.36( \pm .64)$ & $2.28( \pm .83)$ & 0.02 & \\
\hline Physically hurt ${ }^{\dagger}$ & 41.2 & 75.0 & 51.4 & 54.1 & & 0.18 \\
\hline Routine disrupted & 17.6 & 37.5 & 22.2 & 18.1 & & 0.13 \\
\hline Current mental health" & $26.1( \pm 8.4)$ & $22.4( \pm 8.6)$ & $24.4( \pm 7.6)$ & $27.9( \pm 7.3)$ & 0.05 & \\
\hline \multicolumn{7}{|l|}{ Physical assault by caregiver $(n=132)$} \\
\hline Fear & $2.10( \pm .75)$ & $2.20( \pm .84)$ & $2.00( \pm .82)$ & $2.34( \pm .82)$ & 0.03 & \\
\hline Physically hurt & 41.0 & 75.0 & 40.9 & 51.5 & & 0.14 \\
\hline Routine disrupted & 15.4 & 20.0 & 18.2 & 18.5 & & 0.04 \\
\hline Current mental health & $25.1( \pm 6.9)$ & $28.2( \pm 7.5)$ & $25.0( \pm 7.0)$ & $26.9( \pm 8.5)$ & 0.02 & \\
\hline \multicolumn{7}{|l|}{ Emotional abuse by caregiver $(n=160)$} \\
\hline Fear & $1.77( \pm .63)$ & $2.08( \pm .87)$ & $1.93( \pm .74)$ & $2.01( \pm .82)$ & 0.02 & \\
\hline Routine disrupted & 10.3 & 0.0 & 10.0 & 5.8 & & 0.11 \\
\hline Current mental health & $26.7( \pm 7.5)$ & $23.2( \pm 9.2)$ & $25.9( \pm 7.7)$ & $24.9( \pm 7.8)$ & 0.02 & \\
\hline \multicolumn{7}{|l|}{ Sexual victimization } \\
\hline \multicolumn{7}{|l|}{ Sexual assault by anyone $(n=44)$} \\
\hline Fear & $2.25( \pm .87)$ & $2.50( \pm .58)$ & $2.67( \pm .52)$ & $2.68( \pm .57)$ & 0.08 & \\
\hline Physically hurt & 25.0 & 66.7 & 0.0 & 42.9 & & 0.37 \\
\hline Routine disrupted & 41.7 & 0.0 & 33.3 & 36.4 & & 0.24 \\
\hline Current mental health & $24.7( \pm 6.2)$ & $23.5( \pm 9.9)$ & $20.5( \pm 11.4)$ & $26.7( \pm 8.0)$ & 0.07 & \\
\hline
\end{tabular}

Note. $n$ varies because only participants who reported that bystanders were present were asked about bystander actions. "No impact" indicates bystander neither helped nor harmed the situation. Fear ratings were on a scale from 1 (not afraid) to 3 (very afraid). Injury was only asked about physical and sexual assault. Percentages or means with outcomes significantly better than expected by chance are in bold (lower fear, injury, and routine disrupted and higher mental health). Percentages or means with outcomes significantly worse than expected by chance are italicized (higher fear, injury and routine disrupted and lower mental health).

${ }^{\dagger} p<.10 .{ }^{*} p<.05 .{ }^{* *} p<.01 .{ }^{* *} p<.001$.

Table 4: Four Outcomes of Victimization as a Function of Bystanders'Actions for 10 Victimization Types

\begin{tabular}{|l|l|l|l|l|l|l|}
\hline & & $\begin{array}{l}\text { Bystander's } \\
\text { actions }\end{array}$ & & & & \\
\hline Victimization type & Helped & Harmed & $\begin{array}{l}\text { Helped \& } \\
\text { harmed }\end{array}$ & No impact & $\eta 2$ & $r$ \\
\hline Peer-perpetrated victimization & & & & & & \\
\hline
\end{tabular}




\begin{tabular}{|c|c|c|c|c|c|c|}
\hline $\begin{array}{l}\text { Assault by non-related peer ( } n= \\
532 \text { ) }\end{array}$ & & & & & & \\
\hline Fear (Mean, SD) $)^{* *}$ & $1.54( \pm .63)$ & $1.92( \pm .73)$ & $1.74( \pm .64)$ & $1.67( \pm .73)$ & 0.02 & \\
\hline Physically hurt (\% yes) & $26.6 \%$ & $42.9 \%$ & $29.3 \%$ & $33.7 \%$ & & 0.10 \\
\hline Routine disrupted (\% yes) ${ }^{* *}$ & $7.2 \%$ & $28.6 \%$ & $8.6 \%$ & $13.0 \%$ & & 0.17 \\
\hline $\begin{array}{l}\text { Current mental health (Mean, } \\
S D)^{*}\end{array}$ & $28.4( \pm 7.4)$ & $24.1( \pm 8.0)$ & $25.9( \pm 6.7$ & ) $27.9( \pm 7.7)$ & 0.02 & \\
\hline \multicolumn{7}{|l|}{ Assault by youth relative $(n=365)$} \\
\hline Fear $^{* *}$ & $1.59( \pm .72)$ & $1.83( \pm .82)$ & $1.70( \pm .67)$ & $1.90( \pm .79)$ & 0.04 & \\
\hline Physically hurt ${ }^{*}$ & 23.9 & 45.8 & 27.3 & 36.2 & & 0.15 \\
\hline Routine disrupted $^{* * *}$ & 6.5 & 39.1 & 4.8 & 14.6 & & 0.27 \\
\hline Current mental health & $27.3( \pm 7.1)$ & $24.3( \pm 7.7)$ & $28.2( \pm 7.0)$ & $26.0( \pm 7.7)$ & 0.02 & \\
\hline \multicolumn{7}{|l|}{$\begin{array}{l}\text { Physical intimidation by peer }(n= \\
453)\end{array}$} \\
\hline Fear $^{* * *}$ & $1.71( \pm .70)$ & $2.11( \pm .76)$ & $1.81( \pm .66)$ & $1.98( \pm .76)$ & 0.04 & \\
\hline Routine disrupted & $10.3 \%$ & $20.0 \%$ & $19.0 \%$ & $14.6 \%$ & & 0.10 \\
\hline Current mental health $^{* * *}$ & $28.26( \pm 6.98)$ & $25.30( \pm 7.35)$ & $22.98( \pm 8.00)$ & $27.00( \pm 7.97)$ & & 0.05 \\
\hline \multicolumn{7}{|l|}{$\begin{array}{l}\text { Relational aggression by peer }(n= \\
527)\end{array}$} \\
\hline Fear ${ }^{*}$ & $1.61( \pm .67)$ & $1.81( \pm .71)$ & $1.84( \pm .64)$ & $1.73( \pm .72)$ & 0.02 & \\
\hline Routine disrupted $^{* * *}$ & 7.1 & 30.2 & 17.5 & 15.9 & & 0.19 \\
\hline Current mental health $^{* *}$ & $27.7( \pm 7.2)$ & $24.5( \pm 8.2)$ & $24.3( \pm 7.9)$ & $26.8( \pm 7.8)$ & 0.03 & \\
\hline \multicolumn{7}{|l|}{$\begin{array}{l}\text { Social discrediting by peer }(n= \\
631)\end{array}$} \\
\hline Fear $^{* * *}$ & $1.35( \pm .57)$ & $1.73( \pm .75$ & ) $1.73( \pm .67)$ & $1.54( \pm .66)$ & 0.06 & \\
\hline Routine disrupted $^{* * *}$ & 6.6 & 33.8 & 20.0 & 12.3 & & 0.24 \\
\hline Current mental health $^{*}$ & $28.4( \pm 7.1)$ & $25.6( \pm 8$ & 3) $26.5( \pm 7.3)$ & $27.1( \pm 7.9)$ & 0.02 & \\
\hline \multicolumn{7}{|l|}{ Social exclusion by peer $(n=536)$} \\
\hline Fear $^{+}$ & $1.33( \pm .56)$ & $1.48( \pm .60)$ & $1.54( \pm .66)$ & $1.44( \pm .60)$ & 0.02 & \\
\hline Routine disrupted & 4.9 & 12.2 & 11.0 & 8.3 & & 0.09 \\
\hline Current mental health & $27.5( \pm 7.0)$ & $24.5( \pm 8.3)$ & $26.2( \pm 7.6)$ & $26.8( \pm 7.4)$ & 0.01 & \\
\hline \multicolumn{7}{|l|}{ Adult-perpetrated victimization } \\
\hline \multicolumn{7}{|l|}{$\begin{array}{l}\text { Physical assault by any adult }\left(n_{-}\right. \\
206)\end{array}$} \\
\hline Fear & $2.10( \pm .79)$ & $2.31( \pm .79)$ & $2.36( \pm .64)$ & $2.28( \pm .83)$ & 0.02 & \\
\hline Physically hurt ${ }^{\dagger}$ & 41.2 & 75.0 & 51.4 & 54.1 & & 0.18 \\
\hline Routine disrupted & 17.6 & 37.5 & 22.2 & 18.1 & & 0.13 \\
\hline Current mental health ${ }^{*}$ & $26.1( \pm 8.4)$ & $22.4( \pm 8.6)$ & $24.4( \pm 7.6)$ & $27.9( \pm 7.3)$ & 0.05 & \\
\hline \multicolumn{7}{|l|}{$\begin{array}{l}\text { Physical assault by caregiver ( } n= \\
132 \text { ) }\end{array}$} \\
\hline Fear & $2.10( \pm .75)$ & $2.20( \pm .84)$ & $2.00( \pm .82)$ & $2.34( \pm .82)$ & 0.03 & \\
\hline Physically hurt & 41.0 & 75.0 & 40.9 & 51.5 & & 0.14 \\
\hline Routine disrupted & 15.4 & 20.0 & 18.2 & 18.5 & & 0.04 \\
\hline Current mental health & $25.1( \pm 6.9)$ & $28.2( \pm 7.5)$ & $25.0( \pm 7.0)$ & $26.9( \pm 8.5)$ & 0.02 & \\
\hline \multicolumn{7}{|l|}{$\begin{array}{l}\text { Emotional abuse by caregiver }(n= \\
160)\end{array}$} \\
\hline Fear & $1.77( \pm .63)$ & $2.08( \pm .87)$ & $1.93( \pm .74)$ & $2.01( \pm .82)$ & 0.02 & \\
\hline Routine disrupted & 10.3 & 0.0 & 10.0 & 5.8 & & 0.11 \\
\hline Current mental health & $26.7( \pm 7.5)$ & $23.2( \pm 9.2)$ & $25.9( \pm 7.7)$ & $24.9( \pm 7.8)$ & 0.02 & \\
\hline \multicolumn{7}{|l|}{ Sexual victimization } \\
\hline \multicolumn{7}{|l|}{ Sexual assault by anyone $(n=44)$} \\
\hline Fear & $2.25( \pm .87)$ & $2.50( \pm .58)$ & $2.67( \pm .52)$ & $2.68( \pm .57)$ & 0.08 & \\
\hline Physically hurt & 25.0 & 66.7 & 0.0 & 42.9 & & 0.37 \\
\hline Routine disrupted & 41.7 & 0.0 & 33.3 & 36.4 & & 0.24 \\
\hline
\end{tabular}


Note. $n$ varies because only participants who reported that bystanders were present were asked about bystander actions. "No impact" indicates bystander neither helped nor harmed the situation. Fear ratings were on a scale from 1 (not afraid) to 3 (very afraid). Injury was only asked about physical and sexual assault. Percentages or means with outcomes significantly better than expected by chance are in bold (lower fear, injury, and routine disrupted and higher mental health). Percentages or means with outcomes significantly worse than expected by chance are italicized (higher fear, injury and routine disrupted and lower mental health).

$+p<.10 .^{*} p<.05 .{ }^{* *} p<.01 .^{* * *} p<$

Our hypothesis regarding bystander actions and victim outcomes was partially supported. For all peer victimizations except social exclusion, victim's fear levels were significantly lower during the incident when the bystander helped the situation, compared with at least one other bystander action $(p<.01$ $-p<.05$; harmed, helped and harmed, or no impact). A similar pattern emerged for disruption of victim's routine: four peer-perpetrated incident types, including assault by youth relatives $(p<.001)$ or nonrelated peers $(p<.01)$, showed significantly lower rates of routine disruption when the bystander helped the situation and significantly higher rates of routine disruption when any harm was done by bystanders. For peer victimization, victim physical injury was assessed for the two assault types, with significantly lower rates of injury when the bystander helped, compared with when the bystander harmed, for assault by youth relatives $(p<.05)$. For the four peer-perpetrated incident types that showed significant differences, current mental health was worse when any bystander harm was done by the bystander compared to at least one other bystander actions.

There were very few significant differences when analyzing outcomes for sexual victimization and adult-perpetrated victimization, perhaps because of smaller sample sizes. Still, the differences that were found followed a similar pattern to those that emerged for peer victimization: when any harm was done by bystanders of physical assault by adults, victim's current mental health was significantly lower $(p<.05)$. All significant relationships that emerged regarding bystander actions were in the same direction as our hypotheses. Table 4 also presents effect sizes for analyses, which ranged from small to medium.

\section{Bystander Safety and Victim Outcomes}

In addition to assessing the association of bystander actions on victim outcomes, we examined how harm or threats to the bystander are associated with the same four victim outcomes, again using chisquares to compare rates of victim physical injury and routine disruption to bystander safety and using analyses of variance to compare fear levels and current mental health scores to bystander safety (see Table 5). 
Table 5

Four Outcomes of Victimization as a Function of Harm or Threat to Bystander for 10 Victimization Types

\begin{tabular}{|c|c|c|c|c|}
\hline Victimization type & $\begin{array}{l}\text { Bystander harmed/ } \\
\text { threatened }\end{array}$ & $\begin{array}{l}\text { Bystander not } \\
\text { harmed }\end{array}$ & $\eta^{2}$ & $r$ \\
\hline \multicolumn{5}{|l|}{ Peer-perpetrated victimizations } \\
\hline \multicolumn{5}{|c|}{ Assault by nonrelated peer $(n=533)$} \\
\hline Fear (Mean, $S D)^{* *}$ & $1.97( \pm .73)$ & $1.60( \pm .67)$ & 0.02 & \\
\hline Physically hurt (\% yes) $)^{* *}$ & $71.0 \%$ & $27.3 \%$ & & 0.22 \\
\hline Routine disrupted (\% yes) ${ }^{*+*}$ & $38.7 \%$ & $8.6 \%$ & & 0.23 \\
\hline Current mental health & $23.83( \pm 8.10)$ & $27.94( \pm 7.39)$ & 0.02 & \\
\hline \multicolumn{5}{|c|}{ Assault by youth relative $(n=365)$} \\
\hline Fear $^{* *}$ & $2.03( \pm .76)$ & $1.66( \pm .74)$ & 0.02 & \\
\hline Physically hurt ${ }^{* *}$ & 54.5 & 26.4 & & 0.18 \\
\hline Routine disrupted ${ }^{* *}$ & 41.2 & 7.6 & & 0.32 \\
\hline Current mental health & $24.97( \pm 7.40)$ & $27.00( \pm 7.33)$ & 0.01 & \\
\hline \multicolumn{5}{|c|}{ Physical intimidation by peer $(n=457)$} \\
\hline Fear $^{* *}$ & $2.18( \pm .75)$ & $1.84( \pm .72)$ & 0.02 & \\
\hline Routine disrupted ${ }^{* * *}$ & 39.5 & 11.7 & & 0.22 \\
\hline Current mental health ${ }^{*}$ & $23.74( \pm 7.32)$ & $27.09( \pm 7.72)$ & 0.01 & \\
\hline \multicolumn{5}{|c|}{ Relational aggression by peer $(n=526)$} \\
\hline Fear & $2.09( \pm .82)$ & $1.69( \pm .68)$ & 0.02 & \\
\hline Routine disrupted ${ }^{*}$ & 27.8 & 13.6 & & 0.10 \\
\hline Current mental health & $24.78( \pm 8.23)$ & $26.6( \pm 7.76)$ & 0.00 & \\
\hline \multicolumn{5}{|c|}{ Social discrediting by peer $(n=629)$} \\
\hline Fear $^{*+\infty}$ & $1.94( \pm .80)$ & $1.50( \pm .64)$ & 0.02 & \\
\hline Routine disrupted ${ }^{* * *}$ & 39.4 & 12.8 & & 0.17 \\
\hline Current mental health & $25.21( \pm 7.72)$ & $27.37( \pm 7.61)$ & 0.00 & \\
\hline \multicolumn{5}{|l|}{ Social exclusion by peer $(n=530)$} \\
\hline Fear ${ }^{*+*}$ & $2.05( \pm .67)$ & $1.40( \pm .58)$ & 0.05 & \\
\hline Routine disrupted ${ }^{* * *}$ & 38.1 & 6.5 & & 0.23 \\
\hline Current mental health ${ }^{*}$ & $23.15( \pm 7.36)$ & $27.04( \pm 7.29)$ & 0.01 & \\
\hline \multicolumn{5}{|l|}{ Adult-perpetrated victimizations } \\
\hline \multicolumn{5}{|c|}{ Physical assault by any adult $(n=209)$} \\
\hline Fear ${ }^{* *}$ & $2.63( \pm .61)$ & $2.18( \pm .79)$ & 0.04 & \\
\hline Physically hurt ${ }^{* *}$ & 73.3 & 47.2 & & 0.18 \\
\hline Routine disrupted ${ }^{*}$ & 33.3 & 17.6 & & 0.14 \\
\hline Current mental health ${ }^{\dagger}$ & $23.56( \pm 8.80)$ & $26.70( \pm 7.78$ & 0.02 & \\
\hline \multicolumn{5}{|c|}{ Physical assault by caregiver $(n=133)$} \\
\hline Fear $^{* *}$ & $2.64( \pm .73)$ & $2.12( \pm .79)$ & 0.06 & \\
\hline Physically hurt ${ }^{* *}$ & 90.5 & 38.7 & & 0.38 \\
\hline Routine disrupted ${ }^{*}$ & 36.4 & 13.6 & & 0.22 \\
\hline Current mental health & $25.48( \pm 8.82)$ & $26.37( \pm 7.59)$ & 0.00 & \\
\hline \multicolumn{5}{|c|}{ Emotional abuse by caregiver $(n=160)$} \\
\hline Fear $^{* *}$ & $2.50( \pm .86)$ & $1.87( \pm .73)$ & 0.07 & \\
\hline Routine disrupted ${ }^{* * *}$ & 41.2 & 2.8 & & 0.47 \\
\hline Current mental health* & $20.81( \pm 7.68)$ & $26.00( \pm 7.72)$ & 0.04 & \\
\hline \multicolumn{5}{|l|}{ Sexual victimization } \\
\hline \multicolumn{5}{|l|}{ Sexual assault by anyone $(n=45)$} \\
\hline Fear & $2.50( \pm .76)$ & $2.51( \pm .69)$ & 0.00 & \\
\hline Physically hurt* & 62.5 & 25.7 & & 0.31 \\
\hline Routine disrupted & 50.0 & 29.7 & & 0.16 \\
\hline Current mental health & $20.71( \pm 7.04)$ & $25.68( \pm 8.24)$ & 0.05 & \\
\hline
\end{tabular}

Note. Fear ratings were on a scale from 1 (not afraid) to 3 (very afraid). Injury was only asked about physical and sexual assault. Participants who reported that no bystanders were present for a victimization type were not asked about harm and threats towards bystanders for that victimization type. Percentages or means with outcomes significantly better than expected by chance are in bold (lower fear, injury, and routine disrupted and higher mental health). Percentages or means with outcomes significantly worse than expected by chance are italicized (higher fear, injury and routine disrupted and lower mental health).

${ }^{\dagger} p<.10 .{ }^{*} p<.05 .{ }^{\cdots} p<.01 .{ }^{\cdots *} p<.001$. 
Table 5 Four Outcomes of Victimization as a Function of Harm or Threat to Bystander for 10 Victimization Types

\begin{tabular}{|c|c|c|c|c|}
\hline Victimization type & Bystander harmed/threatened & Bystander not harmed & $\eta^{2}$ & $r$ \\
\hline \multicolumn{5}{|l|}{ Peer-perpetrated victimizations } \\
\hline \multicolumn{5}{|c|}{ Assault by nonrelated peer $(n=533)$} \\
\hline Fear $(\text { Mean, } S D)^{* *}$ & $1.97( \pm .73$ & ) $1.60( \pm .67)$ & 0.02 & \\
\hline Physically hurt (\% yes) $)^{* * *}$ & $71.0 \%$ & $27.3 \%$ & & 0.22 \\
\hline Routine disrupted $\left(\%\right.$ yes) ${ }^{* * *}$ & $38.7 \%$ & $8.6 \%$ & & 0.23 \\
\hline Current mental health $^{* *}$ & $23.83( \pm 8.10)$ & $27.94( \pm 7.39)$ & 0.02 & \\
\hline \multicolumn{5}{|l|}{ Assault by youth relative $(n=365)$} \\
\hline Fear $^{* *}$ & $2.03( \pm .76)$ & $1.66( \pm .74)$ & 0.02 & \\
\hline Physically hurt $^{* *}$ & 54.5 & 26.4 & & 0.18 \\
\hline Routine disrupted $^{* * *}$ & 41.2 & 7.6 & & 0.32 \\
\hline Current mental health & $24.97( \pm 7.40)$ & $27.00( \pm 7.33)$ & 0.01 & \\
\hline \multicolumn{5}{|c|}{ Physical intimidation by peer $(n=457)$} \\
\hline Fear $^{* *}$ & $2.18( \pm .75)$ & $1.84( \pm .72)$ & 0.02 & \\
\hline Routine disrupted $^{* * *}$ & 39.5 & 11.7 & & 0.22 \\
\hline Current mental health $^{*}$ & $23.74( \pm 7.32)$ & $27.09( \pm 7.72)$ & 0.01 & \\
\hline \multicolumn{5}{|c|}{ Relational aggression by peer $(n=526)$} \\
\hline Fear $^{* * *}$ & $2.09( \pm .82)$ & $1.69( \pm .68)$ & 0.02 & \\
\hline Routine disrupted $^{*}$ & 27.8 & 13.6 & & 0.10 \\
\hline Current mental health & $24.78( \pm 8.23)$ & $26.6( \pm 7.76)$ & 0.00 & \\
\hline \multicolumn{5}{|l|}{ Social discrediting by peer $(n=629)$} \\
\hline Fear $^{* * *}$ & $1.94( \pm .80)$ & $1.50( \pm .64)$ & 0.02 & \\
\hline Routine disrupted $^{* * *}$ & 39.4 & 12.8 & & 0.17 \\
\hline Current mental health & $25.21( \pm 7.72)$ & $27.37( \pm 7.61)$ & 0.00 & \\
\hline \multicolumn{5}{|l|}{ Social exclusion by peer $(n=530)$} \\
\hline Fear $^{* * *}$ & $2.05( \pm .67)$ & $1.40( \pm .58)$ & 0.05 & \\
\hline Routine disrupted $^{* * *}$ & 38.1 & 6.5 & & 0.23 \\
\hline Current mental health ${ }^{*}$ & $23.15( \pm 7.36)$ & $27.04( \pm 7.29)$ & 0.01 & \\
\hline \multicolumn{5}{|l|}{ Adult-perpetrated victimizations } \\
\hline \multicolumn{5}{|c|}{ Physical assault by any adult $(n=209)$} \\
\hline Fear $^{* *}$ & $2.63( \pm .61$ & ) $2.18( \pm .79)$ & 0.04 & \\
\hline Physically hurt $^{* *}$ & 73.3 & 47.2 & & 0.18 \\
\hline Routine disrupted $^{*}$ & 33.3 & 17.6 & & 0.14 \\
\hline Current mental health $^{+}$ & $23.56( \pm 8.80)$ & $26.70( \pm 7.78$ & 0.02 & \\
\hline \multicolumn{5}{|c|}{ Physical assault by caregiver $(n=133)$} \\
\hline Fear $^{* *}$ & $2.64( \pm .73)$ & $2.12( \pm .79)$ & 0.06 & \\
\hline Physically hurt ${ }^{* * *}$ & 90.5 & 38.7 & & 0.38 \\
\hline Routine disrupted $^{*}$ & 36.4 & 13.6 & & 0.22 \\
\hline Current mental health & $25.48( \pm 8.82)$ & $26.37( \pm 7.59)$ & 0.00 & \\
\hline \multicolumn{5}{|c|}{ Emotional abuse by caregiver $(n=160)$} \\
\hline Fear $^{* *}$ & $2.50( \pm .86)$ & $1.87( \pm .73)$ & 0.07 & \\
\hline Routine disrupted $^{* * *}$ & 41.2 & 2.8 & & 0.47 \\
\hline Current mental health $^{*}$ & $20.81( \pm 7.68)$ & $26.00( \pm 7.72)$ & 0.04 & \\
\hline \multicolumn{5}{|l|}{ Sexual victimization } \\
\hline \multicolumn{5}{|l|}{ Sexual assault by anyone $(n=45)$} \\
\hline Fear & $2.50( \pm .76)$ & $2.51( \pm .69)$ & 0.00 & \\
\hline Physically hurt ${ }^{*}$ & 62.5 & 25.7 & & 0.31 \\
\hline Routine disrupted & 50.0 & 29.7 & & 0.16 \\
\hline Current mental health & $20.71( \pm 7.04)$ & $25.68( \pm 8.24)$ & 0.05 & \\
\hline
\end{tabular}


Note. Fear ratings were on a scale from 1 (not afraid) to 3 (very afraid). Injury was only asked about physical and sexual assault. Participants who reported that no bystanders were present for a victimization type were not asked about harm and threats towards bystanders for that victimization type. Percentages or means with outcomes significantly better than expected by chance are in bold (lower fear, injury, and routine disrupted and higher mental health). Percentages or means with outcomes significantly worse than expected by chance are italicized (higher fear, injury and routine disrupted and lower mental health).

$+p<.10 .^{*} p<.05 .^{* *} p<.01 .^{* * *} p<$

Hypotheses were partially confirmed regarding bystander safety and victim outcomes. Victim fear levels were significantly lower when bystanders were not harmed or threatened, for all incident types except sexual victimization. Similarly, for all victimizations except sexual victimization, rates of routine disruption were significantly lower when bystanders were unharmed. For all five victimizations in which physical injury was assessed, rates of victim injury were significantly lower when the bystander was unharmed. For some incident types, including peer assault $(p<.01)$ and emotional abuse by caregiver $(p<.05)$, better current mental health for the victim was significantly associated with bystanders' safety. All but four comparisons (three of these regarding sexual victimization) showed significant differences that confirmed our hypotheses. Effect sizes ranged from small to medium.

It should be noted that analyses were conducted to investigate whether gender moderated the associations between bystander characteristics and outcomes with few significant effects. Patterns were more of gender similarity than difference.

\section{Discussion}

The main findings of our rural community survey were as follows: (a) bystanders were present for most (approximately 2 of every 3 ) victimizations reported for each victimization type except sexual assault (approximately 1 in 6); (b) bystanders of peer victimization were typically friends or acquaintances and bystanders of family violence are typically family members; (c) more bystanders help than harm, but many do neither; (d) when bystanders help, both short- and long-term victim outcomes may be better; and (e) bystander safety was a strong indicator of better short- and long-term victim outcomes. A strength of the current study is that it explored bystander behavior beyond college campuses and urban environments. Further, the study aimed to work across silos of types of violence to examine patterns of similarity and difference in bystander experiences.

\section{Bystanders: How Common and Who They Are}

Violence is sometimes described as occurring "behind closed doors," but that does not mean that violence is entirely private, even family violence. All forms of physical and psychological aggression we assessed were witnessed more often than not, concordant with NCVS data on the frequency of witnesses to violent crime (Planty, 2002). This is an interesting finding given our exploratory hypothesis that bystanders might be less available in rural communities. Sexual victimization was the sole exception, which we discuss in more detail below. Not surprisingly, bystanders of victimization at home are typically relatives of the victim, whereas bystanders of peer violence are most commonly other peers. Strangers and police were rarely present in any victimization setting. This is the first study to track these differences across victimization types for the same sample. These data show the importance of understanding the type of victimization when considering promoting bystander involvement. Family members were the most common witnesses for all of the most severe forms of 
victimization, including sexual victimization and most forms of physical assault victimization. The strong association between type of victimization and identity of bystander suggests more could be done in prevention and intervention to teach people these patterns and adapt responses based on the bystander-victim relationship.

\section{The Scarcity of Bystanders to Sexual Assault}

The number of cases of sexual victimization was relatively small (statistically speaking), and thus caution is needed when interpreting findings. Nonetheless, this exploratory study suggests some interesting patterns that should be followed up in future research in samples with greater numbers of sexual assault cases. Sexual assaults showed a markedly lower rate of bystander presence and we suggest this could be a factor in the skepticism many victims face when they disclose, given that another role of bystanders is to corroborate what happened (Campbell \& Johnson, 1997; Du Mont, Miller, \& Myhr, 2003). Perhaps law enforcement personnel, as well as friends or family of the victim, are accustomed to relying on eyewitness accounts, because the majority of other crime is witnessed. However, because most sexual assault occurs in private, it is not reasonable to assume that eyewitnesses will be available. Improving criminal justice responses to sexual assault may need to address this facet of sexual assault crimes to raise awareness of jurors and investigators. It is also important to note that sexual assaults were associated with the highest levels of bystander harm among all the victimization types. In light of the growing number of sexual assault prevention programs that encourage bystanders to intervene, this is important to address. The current research cautions us that there is much we still need to learn about the potential risks that bystanders face in these situations and what strategies can best be used to promote both helpful bystander action and bystander safety.

\section{Bystander Actions and Victim Outcomes}

Across incident types, bystanders helped more often than they harmed the situation, although for no incident were even half the bystanders helpful. Furthermore, rates of doing nothing or doing something that perhaps was ineffective but not harmful (neither helping nor harming the situation) hovered around rates of $30 \%$ to $40 \%$ for most incidents. This is consistent with surveys that find that the majority of potential bystanders indicate that they do not know how to help (GfK Public Affairs \& Corporate Communications Group, 2013; Knowledge Networks, 2011; Opinion Research Corporation, 2006). While it is unfortunate that only a minority of bystanders help the situation, the good news is that in cases where bystanders helped, better short-term and long-term victim outcomes were apparent. In the short-term, victims were less likely to experience physical injury or routine disruption and they tended to be less afraid when bystanders helped. Furthermore, the association between help from bystanders and better current mental health for the victim suggests that help from bystanders may even impart long-term benefits for the victim. This would need to be confirmed with longitudinal or experimental studies.

An interesting finding from the current study is that simply the presence or absence of the bystander was generally not significantly associated with victim outcomes. Rather, it is about what the bystander does or, as discussed below, whether the bystander is harmed. These findings are consistent with our hypotheses and consistent with longstanding implicit assumptions, apparent from the earliest work on bystanders, that efforts to be helpful and giving have a positive impact. 


\section{Bystander Safety and Victim Outcomes}

Recognizing that danger for bystanders could be a reason for their inaction is important, but the implications of bystander safety extend beyond that. In examining four victim outcomes for 10 victimization types, bystander safety was more consistently related to victim outcomes than help from bystanders. In situations where bystanders were harmed or threatened, victims tended to be more afraid, perhaps because they also feared for the bystander or because perpetrators harming multiple victims appear more frightening. Additionally, victims were more likely to be uninjured in situations where bystanders were also safe, a relationship that suggests several possible explanations. It may be that perpetrators are less likely to use injurious violence when in the presence of bystanders who are safe from being targeted-such as older family members or schoolchildren with higher social status. However, it could be that perpetrators who are less inclined to physically hurt victims are also less inclined to be forceful with bystanders. The association between harm or threat to bystanders and disruption of victims' routines may suggest that such incidents make a bigger scene, are more publicized, or that these incidents affect a larger social group or family. Indeed, it is likely that some bystanders who are harmed, particularly those who witness victimizations by caregivers, are themselves covictims. Differentiating between bystanders and covictims is important conceptually and empirically. To date, most research on bystander intervention focuses almost solely on the decision process of the bystander and much less on the impact of bystanders on victims or on the bystanders' well-being (Burn, 2009). The current research indicates the importance of the interrelationship between victims and bystanders, which also makes sense given that most bystanders were people that victims knew. The impact of the bystander is not only about what bystanders do but also about whether they are harmed in the process. This suggests that we need to develop and investigate more complex models of helping behavior in the context of interpersonal violence situations.

\section{Bystanders in Rural Settings}

The rural setting of these victimizations should also be acknowledged. Victimization is not a strictly urban problem; it is prevalent in rural environments as well, with bystanders in place who can be engaged to aid victims. Further research on this topic is warranted. For example, a recent study by Edwards et al. (2014) found that bystander helping for relationship violence among young adults in rural communities differed within these communities based on income, with more impoverished rural communities also those with greater helping. Such work reminds us that there is significant variation among communities. A thorough understanding of bystander behavior must do more to look at contextual variables and must investigate bystander behavior in many different settings.

\section{Limitations of the Study}

The limitations to the study should be noted when considering the results. These are self-report data and future efforts to include multiple informants or alternative data sources would be valuable. One particular aspect of this measurement issue is that victims reported both whether the bystander helped or harmed and also reported outcomes. Further research is also needed to unpack and better understand the "helped and harmed" category. Qualitative methods would be interesting to use to more thoroughly explore what victims do find helpful from bystanders across these situations.

Time limitations prevented us from asking details about more than one incident and we did not ask about the age of the bystander, beyond specifying that there were teens or adults. Some especially 
high-risk families may not be easily recruited into surveys. Although our large sample allowed us to collect data on a range of incidents, we had more statistical power for more common victimizations such as relational aggression by peers. Additionally, the sample was collected in a rural, southern part of the U.S. with less racial and ethnic diversity than other areas. It will be important for future research to study bystander patterns in diverse communities. While acknowledging these limitations, we note that these data provide some of the most detailed available information on bystander involvement in victimization and some of the first data on the associations of bystander patterns with victim outcomes.

\section{Research Implications}

The relatively uncharted territory of victim outcomes in relation to bystander action or bystander safety has clearer prevention and intervention implications than merely examining bystander presence alone. The field would benefit from further work in this area, especially in the understudied area of bystander intervention for family violence. Other studies and researchers might look at other victimization types or settings. Qualitative research methods could be used to unpack these responses and gain a better understanding of the complexity of the role of bystanders in interpersonal violence victimization situations. We adopted measures of bystander impact from the NCVS, but recommend that future work expand the level of detail on bystander involvement and impact. For example, the response option "the bystander both helped and harmed" may be ambiguous. This option could apply to situations where one bystander aided the victim while another harmed the victim, but participants who reported this may have been referring to one bystander who did both. Further research would benefit from changing the wording or possibly from dividing the "both helped and harmed" category into two options. Further work could benefit from attention to the characteristics of bystanders and whether character strengths can be promoted that would increase the likelihood of being helpful when victimization is witnessed. Our data show that bystanders are seldom strangers. This implies that understanding the broader relationship and relational networks could be an important avenue for future research. Peer networks can promote or discourage aggression (Swartout, 2013) and the events that transpire when victimization is actually witnessed probably emerge from longstanding relationship patterns. Finally, patterns of similarities and differences related to bystander presence across forms of violence helps support arguments for bridging fields of study to better understand the role of bystanders and victimization (Hamby \& Grych, 2013).

The current study focused on bystanders to crimes that took place. Given the recent focus on bystander action as a source of potential crime prevention, future studies should also investigate bystander presence when someone felt they were at risk for becoming a victim, but the incident was prevented. Measuring these sorts of incidents are complicated but an important facet of understanding more fully the roles of bystanders and violence.

\section{Prevention, Clinical, and Policy Implications}

Although it is good news that more bystanders help than harm, and that their help may have lasting benefits for the victim, the large percentage who neither help nor harm is concerning. Bystanders witnessed most victimizations, yet for most victimization types they aided the victim in fewer than half of those witnessed incidents. In part, this may because bystanders do not know how to effectively help others. For example, research conducted by Knowledge Networks (2011) found that the majority of 
polled college students did not know what to do to take helpful action in sexual assault situations. However, another critical reason bystanders may be hesitant to help the victim may be that they risk becoming victims as well. Of the victimization types examined, sexual assault showed one of the lowest rates of help from bystanders (only $28 \%$ ) and by far the highest rate of harmed or threatened bystanders (18\%). Efforts in educating bystanders to intervene on behalf of victims should not only encourage them to help, but should train them to stay safe as well. It should also be acknowledged that some bystanders who do nothing may be victims alongside the primary victim; their own suffering may be inhibiting their ability to help others. On the other hand, some of these bystanders may also be accomplices to the perpetrators. Prevention efforts aimed at families should emphasize victimization in the home, including neglect and verbal abuse; prevention efforts in schools or peer-group settings should emphasize aiding other peers and should address verbal and emotional abuse as well. These programs need to build bystander confidence and teach specific helping skills across a variety of situations. These data support the idea that a helpful bystander can make a positive difference in the lives of victims.

\section{References}

Baldry, A. C. (2003). Bullying in schools and exposure to domestic violence. Child Abuse \& Neglect, 27, 713-732. 10.1016/S0145-2134(03)00114-5

Banyard, V. L. (2008). Measurement and correlates of prosocial bystander behavior: The case of interpersonal violence. Violence and Victims, 23, 83-97. 10.1891/0886-6708.23.1.83

Banyard, V. L. (2011). Who will help prevent sexual violence: Creating an ecological model of bystander intervention. Psychology of Violence, 1, 216-229. 10.1037/a0023739

Banyard, V. L., Moynihan, M. M., Cares, A. C., \& Warner, R. (2014). How do we know if it works? Measuring outcomes in bystander-focused abuse prevention on campuses. Psychology of Violence, 4, 101-115. 10.1037/a0033470

Banyard, V. L., Plante, E. G., \& Moynihan, M. M. (2004). Bystander education: Bringing a broader community perspective to sexual violence prevention. Journal of Community Psychology, 32, 6179. $10.1002 /$ jcop.10078

Briere, J. (1996). Trauma Symptom Checklist for Children (TSCC): Professional manual. Odessa, FL: Psychological Assessment Resources.

Burn, S. M. (2009). A situational model of sexual assault prevention through bystander intervention. Sex Roles, 60, 779-792. 10.1007/s11199-008-9581-5

Campbell, R., \& Johnson, C. R. (1997). Police officers' perceptions of rape: Is there consistency between state law and individual beliefs?Journal of Interpersonal Violence, 12, 255-274.

$10.1177 / 088626097012002007$

Corcoran, J. (2004). Treatment outcome research with the non-offending parents of sexually abused children: A critical review. Journal of Child Sexual Abuse, 13, 59-84. 10.1300/J070v13n02_04

DeBoard-Lucas, R., \& Grych, J. H. (2011). Children's perceptions of intimate partner violence: Causes, consequences, and coping. Journal of Family Violence, 26, 343-354. 10.1007/s10896-011-9368-2

Du Mont, J., Miller, K.-L., \& Myhr, T. L. (2003). The role of "real rape" and "real victim" stereotypes in the police reporting practices of sexually assaulted women. Violence Against Women, 9, 466486. $10.1177 / 1077801202250960$ 
Edwards, K. M., Mattingly, M. J., Dixon, K. J., \& Banyard, V. L. (2014). Community matters: Intimate partner violence among rural young adults. American Journal of Community Psychology, 53, 198-207. 10.1007/s10464-014-9633-7

Elliott, A. N., \& Carnes, C. N. (2001). Reactions of nonoffending parents to the sexual abuse of their child: A review of the literature. Child Maltreatment, 6, 314-331.

10.1177/1077559501006004005

Finkelhor, D., Hamby, S., Ormrod, R., \& Turner, H. (2005). The Juvenile Victimization Questionnaire: Reliability, validity, and national norms. Child Abuse \& Neglect, 29, 383-412.

10.1016/j.chiabu.2004.11.001

Finkelhor, D., Turner, H., Ormrod, R., \& Hamby, S. (2009). Violence, abuse, and crime exposure in a national sample of children and youth. Pediatrics, 124, 1411-1423. 10.1542/peds.2009-0467

Fischer, P., Krueger, J. I., Greitemeyer, T., Vogrincic, C., Kastenmüller, A., Frey, D., . . Kainbacher, M. (2011). The bystander-effect: A meta-analytic review on bystander intervention in dangerous and non-dangerous emergencies. Psychological Bulletin, 137, 517-537. 10.1037/a0023304

GfK Public Affairs and Corporate Communications Group (Producer). (2013). The NO MORE study: Teens and young adults on dating violence and sexual assault. Retrieved from http://www.ncdsv.org/images/GFK No More Study Results 1-2013.pdf

Hamby, S., Finkelhor, D., Ormrod, R., \& Turner, H. (2004). The Juvenile Victimization Questionnaire (JVQ): Administration and scoring manual. Durham, NH: Crimes Against Children Research Center.

Hamby, S., Finkelhor, D., \& Turner, H. (in press). Intervention following family violence: Best practices $\&$ helpseeking obstacles in a nationally representative sample of families with children. Psychology of Violence.

Hamby, S., Finkelhor, D., Turner, H., \& Ormrod, R. (2011). Children's exposure to intimate partner violence and other family violence (NCJ232272). Washington, DC: U. S. Department of Justice.

Hamby, S., \& Grych, J. (2013). The web of violence: Exploring connections among different forms of interpersonal violence and abuse. New York, NY: Springer. 10.1007/978-94-007-5596-3

Hart, T. C., \& Miethe, T. D. (2008). Exploring bystander presence and intervention in nonfatal violent victimization: When does helping really help?Violence and Victims, 23, 637-651. 10.1891/08866708.23.5.637

Hartley, C. C. (2002). The co-occurrence of child maltreatment and domestic violence: Examining both neglect and child physical abuse. Child Maltreatment, 7, 349-358. 10.1177/107755902237264

Knowledge Networks(Producer). (2011, January10, 2013). 2011 College dating violence and abuse poll report [Press Release]. Retrieved from http://www. loveisrespect.org/pdf/College Dating And Abuse Final Study.pdf

Latané, B., \& Darley, J. M. (1968). Group inhibition of bystander intervention in emergencies. Journal of Personality and Social Psychology, 10, 215-221. 10.1037/h0026570

Lodge, J., \& Frydenberg, E. (2005). The role of peer bystanders in school bullying: Positive steps toward promoting peaceful schools. Theory into Practice, 44, 329-336. 10.1207/s15430421tip4404_6

Nickerson, A. B., Mele, D., \& Princiotta, D. (2008). Attachment and empathy as predictors of roles as defenders or outsiders in bullying interactions. Journal of School Psychology, 46, 687-703. 10.1016/j.jsp.2008.06.002 
Oh, I., \& Hazler, R. J. (2009). Contributions of personal and situational factors to bystanders' reactions to school bullying. School Psychology International, 30, 291-310. 10.1177/0143034309106499

Opinion Research Corporation. (2006). Bystander survey.

Orchowski, L. M., Gidycz, C. A., \& Raffle, H. (2008). Evaluation of a sexual assault risk reduction and self-defense program: A prospective analysis of a revised protocol. Psychology of Women Quarterly, 32, 204-218. 10.1111/j.1471-6402.2008.00425.x

Planty, M. (2002). Bureau of Justice Statistics Special Report: Third-party involvement in violent crime, 1993-99. Washington, DC: U. S. Department of Justice, Office of Justice Programs.

Rennison, C. M., Dragiewicz, M., \& DeKeseredy, W. S. (2013). Context matters: Violence against women and reporting to police in rural, suburban and urban areas. American Journal of Criminal Justice, 38, 141-159. 10.1007/s12103-012-9164-4

Rushton, J. P. (1978). Urban density and altruism: Helping strangers in a Canadian city, suburb, and small town. Psychological Reports, 43, 987-990. 10.2466/pr0.1978.43.3.987

Salmivalli, C. (2010). Bullying and the peer group: A review. Aggression and Violent Behavior, 15, 112120. 10.1016/j.avb.2009.08.007

Salmivalli, C., Voeten, M., \& Poskiparta, E. (2011). Bystanders matter: Associations between reinforcing, defending, and the frequency of bullying behavior in classrooms. Journal of Clinical Child and Adolescent Psychology, 40, 668-676. 10.1080/15374416.2011.597090

Shernock, S. (2005). Third party roles in intimate partner violence incidents and their effects on police response in a statewide rural jurisdiction. Journal of Police and Criminal Psychology, 20, 22-39. 10.1007/BF02806704

Swartout, K. M. (2013). The company they keep: How peer networks influence male sexual aggression. Psychology of Violence, 3, 157-171. 10.1037/a0029997

Swearer, S. M., Espelage, D. L., Vaillancourt, T., \& Hymel, S. (2010). What can be done about school bullying? Linking research to educational practice. Educational Researcher, 39, 38-47. 10.3102/0013189X09357622 